\title{
Labyrinthe
}

$30 \mid 2008$ (2)

Ecologie $=\mathbf{X}$

\section{Vers une notion écologique du paysage}

Pour une réconciliation des structures naturelles et de l'espace anthropique

Loïc Fel

\section{OpenEdition}

Journals

Édition électronique

URL : https://journals.openedition.org/labyrinthe/3771

DOI : 10.4000/labyrinthe.3771

ISSN : 1950-6031

Éditeur

Hermann

Édition imprimée

Date de publication : 10 juin 2008

Pagination : 61-71

ISBN : 978-2-9526131-7-0

Référence électronique

Loïc Fel, « Vers une notion écologique du paysage », Labyrinthe [En ligne], 30 | 2008 (2), mis en ligne le 27 mai 2008, consulté le 12 janvier 2022. URL : http://journals.openedition.org/labyrinthe/3771 ; DOI : https://doi.org/10.4000/labyrinthe.3771

Propriété intellectuelle 


\section{VERS UNE NOTION ÉCOLOGIQUE DU PAYSAGE \\ Pour une réconciliation des structures naturelles et de l'espace anthropique}

Lö̈c FEL

fel.loic@laposte.net

Créée en $1861^{1}$, la réserve artistique de 1097 hectares en forêt de Fontainebleau constitue le premier espace naturel protégé au monde. Des artistes, dont Camille Corot, Théodore Rousseau, les peintres paysagistes de l'école de Barbizon, ainsi que des écrivains dont Chateaubriand et Victor Hugo, ont défendu un projet de conservation en l'état de cette forêt parce qu'elle présentait un caractère esthétique particulier du fait de la disparité de ses lignes, de ses formes et de ses couleurs. Plus d'un siècle plus tard, le volet «paysage» des permis de construire en France, mis en place par une loi votée en 1993, revenait pareillement à demander que le projet n'entraîne pas de rupture malheureuse dans la vue d'ensemble du site, quelles qu'en soit les qualités bio-environnementales. Aujourd'hui encore, lorsque le paysage est pris en compte dans les programmes d'aménagement du territoire ou en urbanisme, il est souvent d'abord conçu comme un espace anthropique sous la forme d'un cadre de vie, une identité culturelle ou sociale, avant d'être perçu en tant qu'environnement naturel.

Les politiques de création d'espaces naturels protégés initiées depuis un demi-siècle se sont en revanche largement fondées sur des enjeux écologiques - au sens scientifique du terme - jusqu'à substituer aux réserves artistiques des réserves biologiques. Les secondes apparaissent en 1953, une petite vingtaine d'années avant la disparition des premières, en 1974. Aujourd'hui, la forêt de Fontainebleau cumule différents statuts: membre du réseau Natura 2000, Réserve de biosphère (depuis 1998), et "forêt de protection» (depuis 2002). En outre, certaines de ses parcelles sont classées Réserves biologiques intégrales, et

1. Loi du 13 aôt 1861 sur la conservation et l'entretien de la forêt de Fontainebleau. 
d'autres Réserves biologiques dirigées. On remarquera que pas un seul intitulé des titres de protection de sites naturels ne contient le terme «paysage».

Récemment, les plans d'aménagement du territoire ont eux aussi intégré la valeur biologique des espaces considérés. Ainsi, à la suite des négociations menées dans le cadre du Grenelle de l'environnement en octobre 2007, et sous l'influence des organisations de protection de la nature, il a été proposé d'ajouter une "trame verte» aux obligations qui accompagnent de tels projets, afin de les inscrire dans une continuité écologique territoriale. Cette trame, fondée sur des critères biogéographiques, serait le pendant scientifique du volet «paysage» de la loi de 1993 qui insistait jusqu'ici sur la continuité visuelle de l'environnement ${ }^{2}$.

La dualité des critères entre lesquels balancent historiquement et conceptuellement les politiques de protection du paysage, peut-elle dès lors être perçue comme le signe d'une dualité inhérente à son concept de base? Ou témoigne-t-elle de la transformation de ce concept par une accumulation de caractéristiques au long de l'histoire? Dans ce cas, un conflit entre l'approche esthétique traditionnelle et celle assise sur des connaissances scientifiques est-elle possible, au point d'invalider la seconde, historiquement plus récente, au profit d'une autre conception (écologique, voire «écolo-éthique»)?

\section{Le paysage, entre esthétique et sciences de la nature}

La notion de paysage peut être entendue comme désignant un espace homogène, perçu en un seul regard, et donnant sur l'horizon. Alain Roger propose de penser le paysage par opposition au pays. Le pays désigne un espace identique au paysage, mais dans une acception fonctionnelle et identitaire: c'est l'espace occupé et structuré par un ensemble d'activités (agriculture, élevage, chasse, réserve de bois, etc.). La différence entre l'espace du pays et l'espace du paysage ne tient pas à l'objet perçu, mais à l'interprétation qu'en fait l'observateur. Le pays est comme un paysage que l'on saisirait sans unité formelle: il est ce site

2. Il s'agit clairement d'aller au-delà de la simple « culture du paysage » qu'analysait Hélène SoulierPiotrowski dans Labyrinthe, $\mathrm{n}^{\circ}$ 15, 2003, p. $49-68$ (dossier "Territoires: Questions d'images n). 
que l'on perçoit uniquement comme un agrégat d'éléments physiques utiles ou non, ce même site étant - pour être vu en tant que paysage considéré sous ses aspects formels. Ce changement de perception est particulièrement sensible dans le Journal de voyage en Italie de Montaigne:

Delà nous trouvâmes un vallon d'une grande longueur au travers duquel passe la rivière d'Inn, qui va se rendre à Vienne dans le Danube $[\ldots]$. Ce vallon sembloit à $M$. de Montaigne représenter le plus agréable païsage qu'il eust jamais veu?

Étranger au pays visité, Montaigne ne perçoit pas immédiatement l'organisation utilitaire et les ressources de cette vallée pourtant habitée et cultivée. Incapable de saisir l'image du "pays» en face de lui, il saisit plutôt les unités formelles d'un espace épuré de toute utilité et de toute finalité. Le texte de Montaigne, écrit autour de 1580, est l'un des premiers où l'on trouve une occurrence du terme "paysage». Anne Cauquelin et Alain Roger n'ont pas manqué de rapprocher l'apparition de ce terme du contexte de la Renaissance, notamment picturale ${ }^{4}$. Mais alors que le premier fait du paysage peint l'expression d'une réalité perçue par les peintres, la seconde fait de la peinture elle-même l'acte par lequel se constitue l'unité formelle du paysage.

Anne Cauquelin - qui souligne que le terme de "paysage» s'applique aussi bien à la représentation qu'à l'objet de la représentation $^{5}$ - affirme que la valeur de la représentation (la peinture du paysage) rejaillit sur le représenté digne d'être peint (le paysage physique). L'art de la composition picturale unifie des éléments naturels dans la représentation d'un ensemble grâce, notamment, à la perspective qui permet de placer les éléments les uns par rapport aux autres de manière cohérente. Cette conception permet en outre d'étendre la notion de paysage à des représentations intégrant les éléments légendaires ou symboliques qui peuvent accompagner l'appréhension esthétique d'une unité naturelle - les paysages de la forêt de Brocéliande, par exemple, for-

3. Michel de Montaigne, dans Eivres complètes, Gallimard, Paris, 1962, p. 1164.

4. Anne Cauquelin, L'Imvention du paysage, Paris, PUF, 2000; Alain Roger, Court Traité du paysage, Paris, Gallimard, 1978

5. L'argument étymologique employé par Anne Cauquelin va dans ce sens: la nomination de la notion de paysage est absente de l'Antiquité et apparaît à la fin de la Renaissance parce que le paysage luimême est une invention de la représentation artistique de cette époque. 
tement rattachés à la légende arthurienne - qui font de ce dernier la «présentation culturellement instituée de cette nature qui [nous]'enveloppe ${ }^{6}$ ».

Il faut attendre le $\mathrm{XIX}^{\mathrm{e}}$ siècle pour que la perception du paysage s'enrichisse de connaissances scientifiques qui assignent à ce dernier une signification nouvelle dont témoigne l'Essai sur la géographie des plantes (1805) d'Alexander von Humboldt et Aimé Bonpland. En remarquant dans les Andes que la répartition spatiale des espèces diffère selon la latitude, l'altitude et le versant de la montagne, Humboldt en vient à formuler une géographie des plantes, véritable étude des aires de répartition qu'il mettra en lien avec les besoins physiologiques propres à chaque espèce. En procédant ainsi, Humboldt construit une représentation raisonnée du paysage. La dimension esthétique n'en est pas moins présente sous la forme d'une fascination pour la complexité, l'harmonie, l' "individualité» des facteurs constitutifs de ce dernier, et la contemplation du résultat de leur concours - «une autre jouissance est celle que produit le caractère individuel du paysage ${ }^{7} »$.

Mais Humboldt inaugure aussi un type d'analyse qui permet d'énoncer les raisons de son organisation. Le paysage n'est plus une identité hasardeuse, fondée sur des données formelles, comme la forêt ou le bocage, qui s'identifient par contraste avec les zones attenantes. Il ne se réduit pas non plus à ce qu'Alain Roger appelait le "pays", puisqu'il s'agit cette fois d'identifier les organisations naturelles, et non plus la structuration de l'espace selon un point de vue utilitariste. Il constitue désormais une unité biologiquement cohérente, dont les parties sont organisées selon des lois et dont l'ensemble des caractéristiques définit un lieu précis (l'énonciation des associations végétales des forêts des Alpes permet par exemple au botaniste de donner la localisation régionale du paysage décrit).

Conçu comme une entité caractéristique d'un lieu géographique (une région, par exemple), le paysage ne continue pas moins de s'inscrire dans l'immense réseau des représentations culturelles qu'il peut étayer, asseoir ou démentir, et auxquelles l'approche esthétique confère un autre type d'unité. À ce stade, la complémentarité de points de vue esthétique et scientifique semble donc devoir s'imposer face à la tentative de les exclure l'un de l'autre. Les références culturelles ne cesse-

6. Anne Cauquelin, L'Invention du paysage, op. cit., p. 127.

7. Alexander von Humboldt, Cosmos, Paris, Gide et Baudry, 1855, t. I, p. 5. 
ront cependant de devoir s'effacer devant la tentative d'objectivation du paysage par la science, cela de manière croissante parallèlement au développement des sciences écologiques.

\section{Le paysage, une unité écologique perceptible}

La conception scientifique du paysage qui résulte de cette inflexion majeure va profondément modifier notre perception, et donc notre compréhension des enjeux d'aménagement du territoire. Dès 1939, Carl Troll, biogéographe allemand, considère le paysage comme un niveau d'analyse efficace en tant que manifestation des processus écologiques retranscrits dans l'espace ${ }^{8}$. Il en fait une unité biologique, un ensemble délimité d'organismes dont les interrelations forment un écosystème (la forêt de Fontainebleau, par exemple). L'organisation spatiale identifiable qu'est le paysage devient l'indication pertinente d'une organisation écologique dont l'étude passe avant tout par une analyse géographique: ce sont les limites physiques qui définissent le périmètre d'un ensemble naturel cohérent et pertinent pour l'analyse systémique. Ce faisant, Carl Troll réintègre dans l'espace d'analyse les organisations anthropiques, sans revenir sur la distinction originale entre pays et paysage.

Le géographe français contemporain Georges Bertrand définit quant à lui le paysage comme le «résultat de la combinaison dynamique, donc instable, d'éléments physiques, biologiques et anthropiques qui, en réagissant dialectiquement les uns sur les autres, font du paysage un ensemble unique et indissociable en perpétuelle évolution ${ }^{9} »$. L'introduction par Georges Bertrand des déterminants anthropiques dans la conception du paysage fait de celui-ci non pas une organisation figée mais un ensemble dynamique: d'une part, les structures du paysage influent sur les activités anthropiques, par exemple une topographie abrupte empêchera les constructions; d'autre part, les structures de paysages créés, comme les systèmes agraires ou les autoroutes, influent sur les écosystèmes en séparant des populations d'une même espèce ou

8. Carl Troll a notamment développé cette théorie dans Okologische Landschaftsforschung und vergleichende Hochgebirgsforschung. Wiesbaden. Franz Steiner, 1966.

9. Voir « Paysage et géographie physique globale ", Revue de géographie des Pyrénées et du SudOuest, vol. 39, n 3, 1968, p. 249-272. 
en augmentant l'érosion des sols. La compréhension de ces déterminismes ne peut être envisagée que sous un angle temporel. La notion de paysage ne correspond donc plus à la simple définition formelle d'un espace, mais elle inclut la conception de son évolution dans le temps.

La notion de paysage, en ne se limitant plus à des considérations spatiales, met donc à jour les enjeux de l'organisation du territoire: la structure du paysage, en tant que déterminant géographique d'écosystème, définit les conditions abiotiques de l'environnement (cette structure influe-t-elle sur l'humidité? sur la composition et l'évolution des sols? sur la qualité de l'air? etc.), et la viabilité dans le temps des espèces végétales qui le composent, comme celle des espèces animales qu'il accueille. En outre, la complexification structurelle du paysage considéré sous l'angle de la biologie et, a fortiori, de l'écologie rend imprécises, du point de vue de ses objectifs, les catégories traditionnellement issues de l'esthétique pour son analyse. De nouvelles notions sont employées: la «matrice», type d'occupation du sol dominant dans un paysage; les «tâches", zones du même paysage occupées différemment, comme par exemple un bosquet au milieu d'une zone de champs ouvert; les «corridors ", éléments longilignes qui relient des tâches de même nature, comme un réseau bocager qui relie des bosquets et des bois. Ces éléments composent la mosaïque du paysage que l'on peut caractériser en termes d'hétérogénéité.

Cette complexification de l'analyse correspond à des réalités écologiques utiles à l'explication de la répartition de populations sur un territoire, ou de la circulation des nutriments. Par exemple, on ne considère plus le paysage des bocages normands comme un ensemble paysagé avec régularité et composé de lignes vertes et d'aplats de couleur (selon les cultures), mais comme une matrice agricole comprenant des corridors écologiques (les haies qui relient des «tâches»: bosquets et forêts); selon la densité et la continuité du réseau corridors-tâches, le paysage sera plus ou moins riche, suivant un critère écologique (et non plus esthétique) de qualité du paysage.

Les écologues spécialisés dans l'étude des paysages constatent que l'évolution de ceux-ci ne suit pas les modèles théoriques vers un climax stable, c'est-à-dire un état d'équilibre durable de l'écosystème qui a terminé son évolution. Par exemple, on considère que le climax européen correspond à la forêt mixte, terme des successions végétales des roches mères jusqu'à la forêt en passant par les stades de la prairie et 
du sous-bois. Au contraire, ce sont les fluctuations des successions végétales qui marquent la norme statistique, et les perturbations naturelles sont intégrées aux écosystèmes. Le parc du Yellowstone constitue un exemple célèbre à ce titre lorsque le modèle climacique est mis en lien avec la succession des feux naturels. Ce que cet exemple nous permet de comprendre des transformations du paysage, c'est tout simplement qu'un fort niveau d'hétérogénéité assure la possibilité de reprise de la succession végétale. Sur un même site, différentes étapes du schéma de la succession végétale cohabitent, permettant en cas de perturbation de recoloniser un espace modifié. Pour la gestion du patrimoine naturel et des paysages, cette correction du modèle climacique permet d'optimiser et d'adapter les choix d'interventions humaines.

C'est ici que la conception du paysage d'un point de vue écologique se démarque de la conception esthétique classique. L'hétérogénéité du paysage apparaît comme garante de sa bonne santé. Un paysage qui mélange des zones forestières denses, des clairières de ronces et des prairies assure une plus grande pérennité et une plus forte biodiversité qu'un paysage uniforme. À l'inverse, la Belle Nature des peintures de paysage relève toujours du «type» et se caractérise par une uniformité marquée. Cette divergence inclut un besoin d'arbitrage pour l'aménagement du territoire entre une volonté de protection de la nature et donc d'un paysage hétérogène, face à un enjeu social, touristique et souvent identitaire, celui d'un paysage uniforme, esthétique, mais écologiquement pauvre.

Pour échapper à cette contradiction interne de la notion de paysage, nous pourrions être tentés de lui substituer celle d'environnement, plus conforme à la prise en compte des données objectives des écosystèmes et qui, chez un philosophe comme Allen Carlson, permet de concilier le plaisir esthétique et la prise en compte des besoins des écosystèmes:

Quand on conceptualise l'environnement naturel en tant que nature je pense que nous sommes tentés de le considérer comme un objet. Quand on le conceptualise comme un paysage on est sans aucun doute porté à l'envisager comme un décor. En conséquence, peut-être le concept d'environnement naturel est-il préférable ${ }^{10}$.

10. Allen Carlson, Aesthetics and the Environment, Londres, Routledge, 2000. 
Carlson propose ainsi une esthétique objective, c'est-à-dire basée sur les données scientifiques de l'environnement naturel - à l'image d'un Glen Love qui entend fonder l'écocritique sur la biologie $\rightarrow$ "L'Écocritique et la science: vers une consilience ?" $\leftarrow$. Il montre comment la perception esthétique de la nature est affectée par le développement des connaissances biologiques et l'emprise accrue de nos modes de vie sur le vivant. Selon lui, les sciences de la nature ont développé des notions et des concepts utiles à la perception des ensembles naturels. C'est le sens de son analyse écocritique des descriptions du Mississipi chez Mark Twain ${ }^{11}$ : intuitivement, la contemplation du fleuve offre le plaisir des surfaces miroitantes de l'eau, le flux et le reflux de la masse liquide, l'animation des insectes et animaux alentour - tout ce qui forme le paysage des bords du grand fleuve nord-américain. Les notions scientifiques permettent en plus à l'observateur de percevoir la disposition des éléments (eau, arbres, plantes et animaux), et d'en comprendre intuitivement l'organisation au même titre que l'histoire de l'art permet de comprendre la composition d'une œuvre. Vu sous cet angle, le Mississipi offre une composition, au sens fort, objective, et immanente à l'histoire du bassin hydraulique dans lequel le fleuve s'inscrit.

Si Carlson prend acte de l'importance de la culture scientifique diffuse dans la perception des environnements naturels, il ne montre pas le lien qui mène de la reconnaissance des données objectives de l'objet à son appréciation esthétique. Sa notion d'environnement perd en outre la forte connotation spatiale qui permettrait de le lier directement avec les enjeux de l'aménagement du territoire. La notion d'environnement est sans doute efficace quand il s'agit de prendre en compte la prise en compte des enjeux globaux, comme la qualité de l'air ou le changement climatique et les pollutions, mais pas pour comprendre l'influence que peut avoir, sur des sites particuliers, tel ou tel aménagement sur la biodiversité.

\section{Concilier valeur esthétique et efficacité écologique: l'exemple des greenways}

Une réponse aux insuffisances de la notion d'environnement chez Carlson, et surtout à la demande d'une conciliation entre l'expérience esthétique commune et les enjeux écologiques de l'espace anthropique, 
peut être apportée par les greenways. En écologie du paysage, une greenway correspond à un espace linéaire établit le long d'un corridor naturel, tel que la berge d'un grand fleuve, ou un chemin de fer. L'intérêt écologique potentiel de ces greenways, ou «coulées vertes", est qu'elles peuvent faire office de corridor en reliant des espaces de même nature dans la matrice du paysage. Une telle "coulée verte» permet alors le brassage des populations animales comme végétales.

L'efficacité d'une greenway à ce titre dépend de sa largeur, de sa continuité et de sa densité. Toutes n'ont pas un grand intérêt écologique. En effet, les greenways correspondent à un plan d'aménagement du territoire dans lequel se conjuguent la question paysagère d'agrément et la protection du patrimoine naturel. Par exemple, la coulée verte parisienne, aménagée de 1988 à 2000 par les paysagistes Philippe Mathieux et Jacques Vergely, est longue de 3 kilomètres ${ }^{12}$. Elle suit un ancien chemin de fer, comprenant le viaduc des arts. Cette installation atteint le bois de Vincennes depuis la Bastille, mais ne le relie à aucun autre espace naturel. Par ailleurs, sa forme étroite, aérienne et extrêmement horticole en fait un jardin d'agrément qui n'a pas d'autre fin qu'esthétique. Cette installation correspond plus à une «promenade plantée» qu'à une « coulée verte» proprement dite. À l'inverse, la coulée verte du sud parisien, longue de 12 kilomètres, sur le tracé d'une ligne de TGV dans les Hauts-de-Seine et l'Essonne, présente un plus grand intérêt écologique bien qu'elle corresponde également à un aménagement paysagé avec une promenade plantée et des espaces ludiques. Cette voie plus large et plus dense sur une partie de son parcours relie des parcs (Sceaux) à des forêts (Fausses-Repose et Meudon) et des étangs. Au milieu d'une zone densément urbanisée, elle permet de structurer un réseau de bois, de sous-bois et de milieux humides qui, s'ils restaient isolés, ne seraient pas assez vastes pour conserver des populations durables de nombreuses espèces. Sur un plan formel, cette coulée verte est bien moins horticole que la précédente, avec une organisation moins lisible pour le promeneur, et invite à une autre approche de la nature que celle des promenades aménagées pour la contemplation paysagère et les ornements.

Cette seconde greenway a été conçue avec un effort esthétique traditionnel inhérent à tous les parcs publics mais avec, en plus, le souci

12. Hervé Brunon et Monique Mosser, Le Jardin contemporain, Paris, édition Scala, 2006. 
de laisser des espaces libres pour les générations spontanées, organiser l'espace par rapport à un ensemble plus vaste (relier des bois) et conserver une largeur et une densité végétale suffisantes pour qu'elle présente un intérêt écologique. Cet aménagement favorise la biodiversité ensuite valorisée auprès du promeneur citadin qui prend plaisir aux structures paysagères formelles ou symboliques. L'élément esthétique vient ainsi à l'appui d'une biodiversité dont il souligne la singularité, et l'envie de la préserver. L'esthétique devient l'outil d'une éthique de la préservation.

Dans ce contexte, ce que la notion scientifique de paysage a changé n'est pas la valeur esthétique du paysage ou la validité de cette notion, mais le type d'esthétique qui y correspond. Des paysagistes l'ont bien compris et se sont faits les spécialistes de la médiation entre le paysage comme espace culturel et le paysage comme espace naturel. Gilles Clément ${ }^{13}$, par exemple, milite en ce sens avec ses réalisations qui mettent en place une "gestion différenciée» des parcs et jardins, c'est-àdire sans utilisation de produits artificiels, d'essences inadaptées au site et en laissant la place aux générations spontanées, pour composer un paysage riche, à la convergence des représentations de la nature, à la fois maîtrisée et libre.

Cette nouvelle praxis aboutit à une esthétique immédiatement identifiable: on s'éloigne des espaces paysagés à l'ordonnancement géométrique du parc à la française ou du foisonnement des fragiles variétés horticoles du jardin anglais. L'éthique de Gilles Clément propose d'adapter les formes traditionnelles d'aménagement des paysages aux besoins des espaces naturels comme, par exemple, le parc de l'île SaintGermain (Hauts-de-Seine), qui dévoile un paysage reprenant les structures traditionnelles du jardin avec des parterres de fleurs, des bosquets et des chemins, mais dont le dessin est fluidifié par les générations d'espèces autochtones qui restructurent continuellement l'espace. Ce paysage n'est pas un espace anthropique maîtrisé, mais un écosystème dynamique, multiple, dont les qualités esthétiques se déclinent au niveau de la parcelle qui entoure immédiatement le visiteur comme au niveau du paysage dans son ensemble, à l'échelle de l'île au milieu de la Seine en l'occurrence.

13. Le Jardin en mouvement, Paris, Pandora, 1991. 


\section{Conclusion}

En retraçant ainsi l'histoire de la notion de paysage, de son élaboration esthétique à sa critique scientifique, nous comprenons bien comment les politiques d'aménagement du territoire en dépendent - signe, sans doute, du changement actuel de mentalité de la pensée urbanistique et de l'aménagement du territoire. Concevoir le paysage comme l'organisation spatiale résultant des dynamiques de l'écosystème pondérées par les activités anthropiques sans qu'elles s'empêchent les unes les autres, permet de poursuivre une gestion du paysage conforme aux principes du développement durable. Ce modèle répond aux besoins scientifiques et éthiques de protection du patrimoine naturel, tout en permettant de concevoir un espace anthropique qui satisfait les aspirations esthétiques, cela d'autant plus aisément que le goût contemporain se tourne vers une nature spontanée et dynamique en lieu et place de la vision d'une nature idéalisée et figée de la peinture de paysage. Mais sur site, il n'en demeure pas moins délicat de parvenir à un tel consensus en regard des enjeux contradictoires d'une part, et des particularités propres à chaque projet. 